\title{
Multifractality and intermittency in the solar wind
}

\author{
W. M. Macek \\ Faculty of Mathematics and Natural Sciences. College of Sciences, Cardinal Stefan Wyszyński University in Warsaw, \\ Dewajtis 5, 01-815 Warszawa, Poland \\ Space Research Centre, Polish Academy of Sciences, Bartycka 18 A, 00-716 Warszawa, Poland
}

Received: 5 June 2007 - Revised: 1 October 2007 - Accepted: 1 October 2007 - Published: 16 November 2007

\begin{abstract}
Within the complex dynamics of the solar wind's fluctuating plasma parameters, there is a detectable, hidden order described by a chaotic strange attractor which has a multifractal structure. The multifractal spectrum has been investigated using Voyager (magnetic field) data in the outer heliosphere and using Helios (plasma) data in the inner heliosphere. We have also analyzed the spectrum for the solar wind attractor. The spectrum is found to be consistent with that for the multifractal measure of the self-similar one-scale weighted Cantor set with two parameters describing uniform compression and natural invariant probability measure of the attractor of the system. In order to further quantify the multifractality, we also consider a generalized weighted Cantor set with two different scales describing nonuniform compression. We investigate the resulting multifractal spectrum depending on two scaling parameters and one probability measure parameter, especially for asymmetric scaling. We hope that this generalized model will also be a useful tool for analysis of intermittent turbulence in space plasmas.
\end{abstract}

\section{Introduction}

The question of multifractality is of great importance for heliophysics because it allows us to look at intermittent turbulence in the solar wind (e.g., Marsch and Tu, 1997; Bruno et al., 2001). Starting from Richardson's scenario of turbulence, many authors try to recover the observed scaling exponents, using some simple and more advanced models of the turbulence describing distribution of the energy flux between cascading eddies on various scales. In particular, the multifractal spectrum has been investigated using Voyager (magnetic field) data in the outer heliosphere (e.g., Burlaga, 1991,

Correspondence to: W. M. Macek

(macek@cbk.waw.pl)
2001) and using Helios (plasma) data in the inner heliosphere (e.g., Marsch et al., 1996).

A direct determination of the multifractal spectrum from the data is known to be a difficult problem. Indication for a chaotic attractor in the slow solar wind has been given by Macek (1998) and by Macek and Redaelli (2000). In particular, Macek (1998) has calculated the correlation dimension of the reconstructed attractor in the solar wind and has provided tests for this measure of complexity including statistical surrogate data tests (Theiler et al., 1992). Further, Macek and Redaelli (2000) have shown that the Kolmogorov entropy of the attractor is positive and finite, as it holds for a chaotic system.

We have extended our previous research on the dimensional time series analysis (Macek, 1998). Namely, we have applied the technique that allows a realistic calculation of the generalized dimensions of the solar wind flow directly from the cleaned experimental signal by using the Grassberger and Procaccia (1983) method. The resulting spectrum of dimensions shows the multifractal structure of the solar wind in the inner heliosphere (Macek et al., 2005, 2006; Macek, 2006). Using a short data sample, we first demonstrate the influence of noise on these results and show that noise can efficiently be reduced by a singular-value decomposition filter (Macek, 2002, 2003). Using a longer sample we have shown that the multifractal spectrum of the solar wind attractor reconstructed in the phase space is consistent with that for the multifractal measure of the self-similar weighted baker's map corresponding to the Cantor set (Macek et al., 2005; Macek, 2006) and, in particular, with the one-scale weighted Cantor set (Macek et al., 2006).

In order to further quantify the multifractality, we thus consider two-scale weighted Cantor set. We investigate the resulting multifractal spectrum depending on two scaling parameters and one probability measure parameter, especially for asymmetric scaling. We hope that this asymmetric model will be a useful tool for analysis of intermittent turbulence in

Published by Copernicus Publications on behalf of the European Geosciences Union and the American Geophysical Union. 


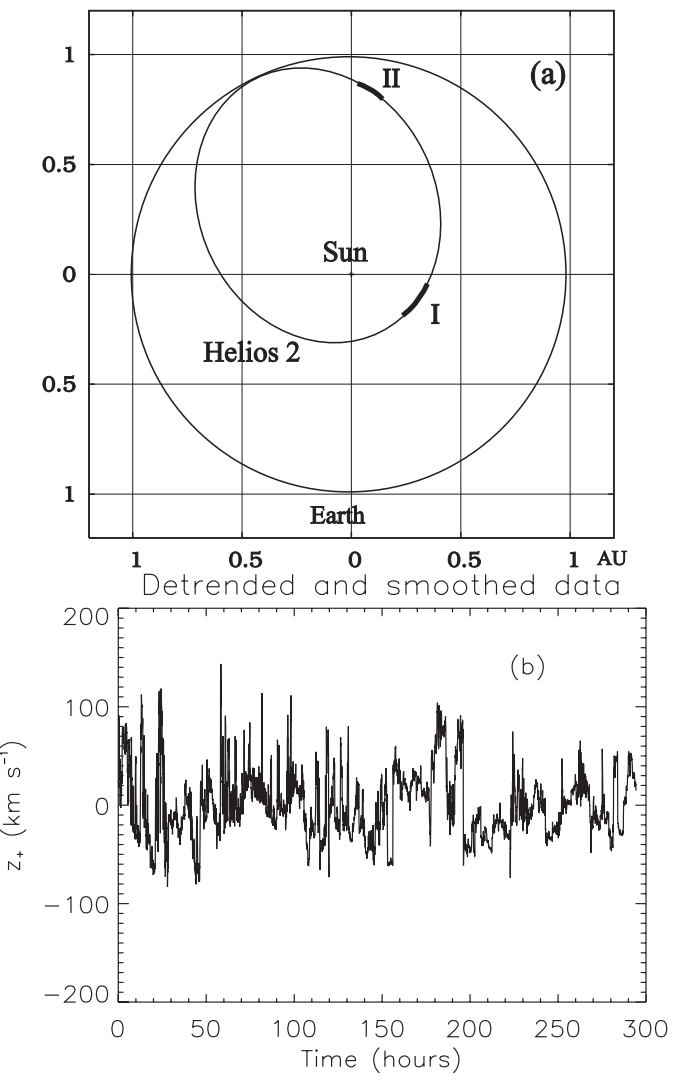

Fig. 1. (a) The orbit of Helios 2 spacecraft, which measured the radial flow velocity with Alfvénic velocity, $v$ and $v_{A}$, in 1977 from 116:00 to 121:21 (day:hour) at distance 0.3 AU and from 348:00 to 357:00 at distance 0.9 AU from the Sun. (b) The Elsässer variable $z_{+}=v \pm v_{A}$ for $B_{o}$ pointing to/away from the Sun for the detrended and filtered data using singular-value decomposition with the five largest eigenvalues.

space plasmas. In particular, taking two different scales for eddies in the cascade, one obtains a more general situation than in the usual $p$-model for fully developed turbulence.

\section{Solar wind data}

In this paper we analyze the Helios 2 data using plasma parameters measured in situ in the inner heliosphere (Schwenn, 1990). The $X$-velocity (mainly radial) component of the plasma flow, $v$, has been investigated by Macek (1998) and by Macek and Redaelli (2000). However, various disturbances are superimposed on the overall structure of the solar wind, including mainly Alfvén waves. Therefore, we take into account Alfvénic fluctuations of the flow. Namely, Macek et al. (2005) analyze the radial $(X$-)component of one of the Elsässer variables, $x=z_{+}$, representing Alfvénic fluctuations propagating outward from the Sun. We have $z_{+}=v+v_{A}$ for the unperturbed magnetic field $B_{o}$ pointing to the Sun and $z_{+}=v-v_{A}$ for $B_{o}$ pointing away from the Sun, where $v_{A}=B /\left(\mu_{o} \rho\right)^{1 / 2}$ is the Alfvénic velocity calculated from the experimental data: the radial component of the magnetic field of the plasma $B$ and the mass density $\rho$ ( $\mu_{o}$ is the permeability of free space).

This analysis focuses on slow wind only and it has been shown that slow wind does not evolve much compared to fast wind (e.g., Tu and Marsch , 1990). Therefore, assuming absence of radial evolution, we have merged two selected time intervals of data separated by about $0.5 \mathrm{AU}$ as measured by the Helios 2 spacecraft in 1977 (i) from 116:00 to 121:21 (day:hour) at distances $0.30-0.34 \mathrm{AU}$ and (ii) from 348:00 to 357:00 at 0.82-0.88 AU from the Sun as shown in Fig. 1a. These raw data of $v$ and $v_{A}, N=26163$ points, with sampling time of $\Delta t=40.5 \mathrm{~s}$, are shown in Fig. 1a of (Macek et al., 2005; Macek, 2006). The first sample of $N=10644$ points have been investigated by Macek et al. (2006).

In Macek et al. (2005) slow trends were subtracted from the original data $v\left(t_{i}\right)$ and $v_{A}\left(t_{i}\right)$, where $i=1, \ldots, N$. The data with the initial several-percent noise level were (eightfold) smoothed (replacing each data point with the average of itself and its two nearest neighbors). Next, the data have been filtered using a method of singular-value decomposition analysis described by Albano et al. (1988). As argued by Macek (1998) we have used five principal eigenvalues. The detrended and filtered data for the radial component of the Elsässer variable $x=z_{+}$are shown in Fig. 1b also taken from Macek et al. (2005).

Table 1 summarizes selected calculated characteristics of the detrended data cleaned by using the singular-value decomposition filter (see, Macek et al., 2005). The probability distributions are clearly non-Gaussian. We have a large skewness of $\sim 0.59$ (as compared with its normal standard deviation 0.02) and a large kurtosis of 0.37 , the latter was small for the analysis with no magnetic field (cf. Macek, 1998). We choose a time delay $\tau=174 \Delta t$, equal to the autocorrelation time $t_{\mathrm{a}}$ where the autocorrelation function decreases to 1/e (cf. Macek, 2003, Fig. 1b). This makes certain that $x(t)$ and $x(t+\tau)$ are at least linearly time independent (e.g., Ott, 1993).

\section{Generalized dimensions}

The generalized dimensions of attractors are important characteristics of complex dynamical systems (e.g., Grassberger, 1983; Hentschel and Procaccia, 1983). Since these dimensions are related to frequencies with which typical orbits in phase space visit different regions of the attractors, they provide information about dynamics of the systems (Ott, 1993). More precisely, one may distinguish a probability measure from its geometrical support, which may or may not have fractal geometry. Then, if the measure has different fractal dimensions on different parts of the support, the measure is multifractal (Mandelbrot, 1989). 
Using our time series of equally spaced, detrended, and cleaned data, we construct many vectors $\mathbf{X}\left(t_{i}\right)=\left[x\left(t_{i}\right), x\left(t_{i}+\tau\right), \ldots, x\left(t_{i}+(m-1) \tau\right)\right]$ in the embedding phase space of dimension $m$, where $i=1, \ldots, n$ with $n=N-(m-1) \tau$. Then, in this space we construct a large number $M(r)$ of hyperspheres of radius $r$ which cover the presumed attractor. If $p_{j}$ is the probability measure that a point from a time series falls in a typical $j$ th hypersphere, using the $q$-order function $I_{q}(r)=\sum\left(p_{j}\right)^{q}, j=1, \ldots, M$, the $q$-order generalized dimension $D_{q}$ is given, e.g., by Ott (1993)

$\tau(q) \equiv(q-1) D_{q}=\lim _{r \rightarrow 0} \frac{\ln I_{q}(r)}{\ln r}$,

where $q$ is a continuous index, $-\infty<q<\infty$. We see from Eq. (1) that the larger $q$ is, the more strongly are the higherprobability spheres (visited more frequently by a trajectory) weighted in the sum for $I_{q}(r)$. Only if $q=0$, all the hyperspheres are counted equally, $I_{0}=M$, and we recover the box-counting dimension, $D_{0}=\lim _{r \rightarrow 0}[\ln M(r) / \ln (1 / r)]$. The limit $q \rightarrow 1$ provides the information dimension, $D_{1}=\lim _{r \rightarrow 0}\left[\sum\left(p_{j} \ln p_{j}\right) / \ln r\right]$.

Writing $I_{q}(r)=\sum p_{j}\left(p_{j}\right)^{q-1}$ as a weighted average $\left\langle\left(p_{j}\right)^{q-1}\right\rangle$, one can associate bulk with the generalized average probability per hypersphere $\mu=\sqrt[q-1]{\left\langle\left(p_{j}\right)^{q-1}\right\rangle}$, and identify $D_{q}$ as a scaling of bulk with size, $\mu \propto r^{D_{q}}$. Since the data cannot constrain well the capacity dimension $D_{0}$, we look for higher-order dimensions, which quantify the multifractality of the probability measure of the attractor. For example, the limit $q \rightarrow 1$ leads to a geometrical average, and the information dimension is $D_{1} \approx\left\langle\ln p_{j}\right\rangle / \ln r$. For $q=2$ the generalized average is the ordinary arithmetic average, with the standard correlation dimension $D_{2} \approx \ln \left\langle p_{j}\right\rangle / \ln r$, and for $q=3$ it is a root-mean-square average. In practice, the probability for a $j$ th hypersphere of radius $r$ is the ratio of the number of distances from a chosen vector $\mathbf{X}\left(t_{j}\right)$ that are less than $r$ to the total number of distances between this vector and other vectors

$p_{j} \simeq \frac{1}{n-2 n_{\mathrm{c}}-1} \sum_{i=n_{\mathrm{c}}+1}^{n} \theta\left(r-\left|\mathbf{X}\left(t_{i}\right)-\mathbf{X}\left(t_{j}\right)\right|\right)$

with $\theta(x)$ being the unit step function, and $n_{\mathrm{c}}$ is the Theiler's (1986) correction ( $n_{\mathrm{c}}=4$ is chosen). Finally, for a given $m$, $I_{q}(r)$ is taken to be equal to the generalized $q$-point correlation sum (Grassberger and Procaccia, 1983)

$C_{q, m}(r)=\frac{1}{n_{\mathrm{ref}}} \sum_{j=1}^{n_{\mathrm{ref}}}\left(p_{j}\right)^{q-1}$

where $n_{\text {ref }}$ is the number of reference vectors $\left(n_{\text {ref }}=5000\right.$ is taken). For large dimensions $m$ and small distances $r$ in the scaling region it can be argued that $C_{q, m}(r) \propto r^{\tau(q)}$, where $\tau(q)$ is an approximation of the ideal limit $r \rightarrow 0$ in Eq. (1) for a given $q$ (Grassberger and Procaccia, 1983).
Table 1. Solar wind velocity fluctuations ${ }^{1}$ data.

\begin{tabular}{ll}
\hline Number of data points, $N$ & 26163 \\
Sampling time, $\Delta t$ & $40.5 \mathrm{~s}$ \\
Skewness $^{2}, \kappa_{3}$ & 0.59 \\
Kurtosis $^{2}, \kappa_{4}$ & 0.37 \\
Minimum frequency & $9.4 \times 10^{-7} \mathrm{~Hz}$ \\
Dominant frequency & $2.5 \times 10^{-5} \mathrm{~Hz}$ \\
Maximum frequency & $1.2 \times 10^{-2} \mathrm{~Hz}$ \\
Autocorrelation time & $4, t_{\mathrm{a}}$ \\
Correlation dimension $^{5}, D_{2}$ & $7.1 \times 10^{3} \mathrm{~s}$ \\
Entropy $^{3,5},(q=2), K_{2}$ & $3.35 \pm 0.21$ \\
Largest Lyapunov exponent $^{3,5}, \lambda_{\max }$ & 0.1 \\
Predictability horizon time $^{3}$ & $1 / 4-1 / 3$ \\
\hline
\end{tabular}

${ }^{1}$ Slow trends (i) $344.596-20.291 t-0.358 t^{2}$, and 88.608-452.349t+343.471 $t^{2}$

(ii) $397.847-291.602 t-241.999 t^{2}$, and

$-30.050+87.756 t-77.773 t^{2}$ (with $t$ being a fraction of a given sample) were subtracted from the original data, $v\left(t_{i}\right)$ and $v_{A}\left(t_{i}\right)$, in $\mathrm{km} \mathrm{s}^{-1}$, and the data were (8-fold) smoothed using moving averages and singular-value decomposition with five eigenvalues. The resulting range of data $x_{\max }-x_{\min }=226.4 \mathrm{~km} \mathrm{~s}^{-1}$.

2 The third and fourth moments of the distribution function are (with average velocity $\langle x\rangle=0.622 \mathrm{~km} \mathrm{~s}^{-1}$ and standard deviation $\sigma=33.514 \mathrm{~km} \mathrm{~s}^{-1}$ )

$\kappa_{3}=\frac{1}{N} \sum_{i=1}^{N}\left[\frac{x_{i}-<x>}{\sigma}\right]^{3}, \kappa_{4}=\frac{1}{N} \sum_{i=1}^{N}\left[\frac{x_{i}-<x>}{\sigma}\right]^{4}-3$

3 Approximately.

4 The autocorrelation time $t_{\mathrm{a}}$ is given by $\left(\left\langle x(t) x\left(t+t_{\mathrm{a}}\right)\right\rangle-\langle x(t)\rangle^{2}\right) / \sigma^{2}=\mathrm{e}^{-1}$. We take a delay $\tau=t_{\mathrm{a}}=174 \Delta t$, which is smaller than the first zero of this function, $1250 \Delta t$.

5 For large enough $m$, we have a slope of a plateau $(q=2$ in Eq. 1). $D_{2} \approx \frac{\ln C_{m}(r)}{\ln (r)}$, where the correlation function is given in Eq. (3). The average slope for $6 \leq m \leq 10$ is taken as $D_{2}$. Similarly, $K_{2} \approx \frac{1}{3} \log _{2}\left[\frac{C_{m}(r)}{C_{m+3}(r)}\right]$ is the $q=2$ entropy (base 2) in the same units as $\lambda_{\max }$ (bits per data sample), $8 \leq m \leq 10$.

\section{Dimensions and multifractality}

We first calculate the natural logarithm of the generalized correlation sum $C_{q, m}(r)$ of Eq. (3) versus $\ln r$ (normalized) for various $q$ and embedding dimensions (cf. Macek, 2002, Fig. 2). We have verified that for $q>0$ the slopes $D_{q, m}(r)=\mathrm{d}\left[\ln C_{q, m}(r)\right] / \mathrm{d}(\ln r) /(q-1)$ in the scaling region of $\ln r$ do not change substantially with the number of points used, providing that the dimension of the attractor is well below $2 \log _{10} N \approx 9$, for $N=26163$ (Eckmann and Ruelle, 1992). The results obtained using the moving average filter and singular-value decomposition linear filter for standard $q=2$ are given by Macek et al. (2005, Fig. 2), and are compared with $q=-2$ in Figs. $2 \mathrm{a}$ and b of Macek (2006), correspondingly, while those obtained for somewhat shorter 


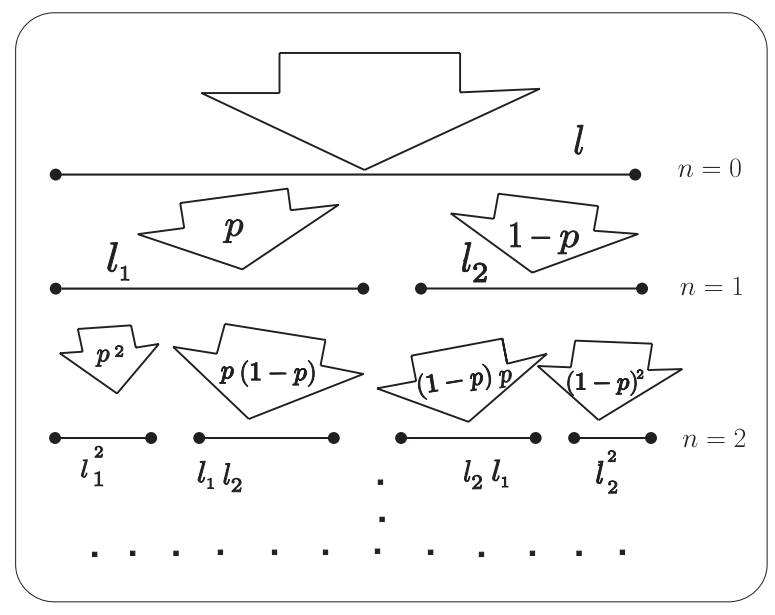

Fig. 2. Generalized two-scale weighted Cantor set model for solar wind turbulence.

samples $(N=4514)$ have been discussed by Macek (1998) and by Macek and Redaelli (2000), using the nonlinear Schreiber filters.

Next, the generalized dimensions $D_{q}$ in Eq. (1) as a function of $q$ with the statistical errors of the average slopes (obtained using weighted least squares fitting) over the scaling range are shown in Fig. 3a (cf. Macek et al., 2005, Fig. 3).

In order to quantify the multifractality we use a simple two-dimensional analytical model of the dynamical system. Namely, we consider the generalized self-similar baker's map acting on the unit square using three parameters $l_{1}, l_{2}$, and $p$ describing nonuniform compression and natural invariant measure of the attractor of the system, correspondingly (e.g., Ott, 1993):

$$
\begin{aligned}
& x_{n+1}= \begin{cases}l_{1} x_{n} & \text { for } y_{n}<p \\
\left(1-l_{2}\right)+l_{2} x_{n} & \text { for } y_{n} \geq p\end{cases} \\
& y_{n+1}= \begin{cases}\frac{y_{n}}{p} & \text { for } y_{n}<p \\
\frac{y_{n}-p}{1-p} & \text { for } y_{n} \geq p\end{cases}
\end{aligned}
$$

where the probability of visiting one region of the square is $p$ (say, $p \leq 1 / 2$ ), and for the remaining region is $1-p$. The parameters $l_{1}$ and $l_{2}$, where $l_{1}+l_{2} \leq 1$ describe both the stretching and folding in the phase space and are related to dissipation (no dissipation for $l_{1}+l_{2}=1$ ). Applying $n$ iterations of Eq. (4) we obtain $\left(\begin{array}{l}n \\ k\end{array}\right)$ vertical strips of width $l_{1}^{m} l_{2}^{n-k}$, where $k=1, \ldots, n$, visited with various probabilities. The resulting strange attractor (of $2^{n}$ strips for $n \rightarrow \infty$ ) is the generalized weighted two-scale Cantor set of narrow strips of various widths and probabilities as schematically shown in Fig. 2. This action of this map exhibits stretching and folding properties leading to sensitive dependence on initial conditions. For the generalized dimensions of the attractor projected onto one axis, for any $q$ in Eq. (1), one obtains $D_{q}=\tau(q) /(q-1)$ by solving numerically the following transcendental equation (e.g., Ott, 1993)

$p^{q} l_{1}^{-\tau(q)}+(1-p)^{q} l_{2}^{-\tau(q)}=1$

The multifractal singularity spectrum $f(\alpha)$ is also obtained from Eq. (5) by the following Legendre transformation

$$
\begin{array}{r}
\alpha(q)=\frac{\mathrm{d}[\tau(q)]}{\mathrm{d} q} \\
f(\alpha)=q \alpha(q)-\tau(q)
\end{array}
$$

When both the stretching and the compression are uniform, $l_{1}=l_{2}=s$, Eq. (5) can be solved explicitly to give the formula for the generalized dimensions of the attractor projected onto one axis (Macek, 2006)

$\tau(q) \equiv(q-1) D_{q}=\frac{\ln \left[p^{q}+(1-p)^{q}\right]}{\ln s}$.

In the absence of dissipation $(s=1 / 2)$ one recovers the formula for the multifractal cascade $p$-model for fully developed turbulence (Meneveau and Sreenivasan, 1987), which obviously corresponds to the weighted one-scale Cantor set (Hentschel and Procaccia, 1983), (cf. Macek, 2002, Fig. 3) and (Macek et al., 2006, Fig. 3b). The difference of the maximum and minimum dimensions, associated with the leastdense and most-dense points on the attractor, correspondingly, is $D_{-\infty}-D_{+\infty}=\ln (1 / p-1) / \ln (1 / s)$ and in the limit $p \rightarrow 0$ this difference rises to infinity. Hence, for a given $s$ the parameter $p$ can be regarded as a degree of multifractality. In particular, the usual middle one-third Cantor set without any multifractality is recovered with $p=1 / 2$ and $s=1 / 3$.

In Fig. 3b we show the singularity spectrum $f(\alpha)$, which follows from Eq. (8) by using Eqs. (6) and (7). It is well known that for $q<0$ the spheres (or cubes) visited less frequently by a trajectory of the system are more important, and we have some basic statistical problems, as seen in Fig. 2b of Macek (2006). Nevertheless, in spite of large statistical errors in Figs. 3a and b, especially for $q<0$, the multifractal character of the measure can still be discerned. Therefore, one can say that the spectrum of dimensions still exhibits the multifractal structure of the slow solar wind in the inner heliosphere.

For illustration the results for $D_{q}+3$ and $f(\alpha)$, fitted to the experimental values of the generalized dimensions with $p=0.12$ and $s=0.47$ in Eq. (8) (see, Macek et al., 2005, Fig. 3), are also shown here by dashed lines in Figs. 3a and $\mathrm{b}$, correspondingly. We expect that intermittency and the degree of multifractality should be stronger for the model with two different scaling parameters. Therefore, a somewhat better fit (for $q<0$ ) to the values of the generalized dimensions is obtained numerically from Eq. (5) for the weighted twoscale Cantor set with an asymmetric scaling using $p=0.20$ and $l_{1}=0.60, l_{2}=0.25$ as is shown in Fig. 4 a by dashed line. The values of the singularity spectrum $f(\alpha)$ projected onto 

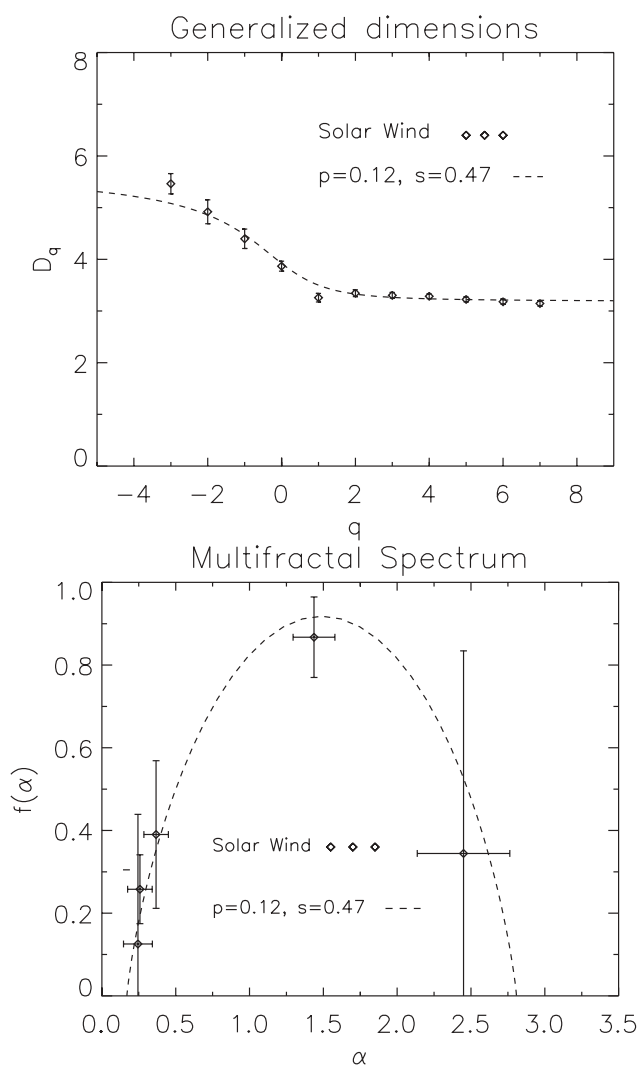

Fig. 3. (a) The generalized dimensions $D_{q}$ in Eq. (1) as a function of $q$. The correlation dimension is $D_{2}=3.4 \pm 0.2$ (see Table 1 ). The values of $D_{q}+3$ are calculated analytically for the weighted baker's map with $p=0.12$ and $s=0.47$ (dashed line). (b) The singularity spectrum $f(\alpha)$ as a function of $\alpha$. The values of $f(\alpha)$ projected onto one axis for the weighted baker's map with the same parameters (dashed), taken from (Macek, 2006).

one axis for the generalized two-scale Cantor set as a function of $\alpha$ with the same parameters are shown by dashed line in Fig. 4b.

We see that the multifractal spectrum of the solar wind is roughly consistent with that for the multifractal measure of the self-similar weighted symmetric baker's map or onescale weighted Cantor set. This spectrum is also in a very good agreement with two-scale asymmetric weighted Cantor set schematically shown in Fig. 2. In particular, taking two different scales for eddies in the cascade, one obtains a more general situation than in the usual $p$-model of Meneveau and Sreenivasan (1987) for fully developed turbulence, especially for an asymmetric scaling, $l_{1} \neq l_{2}$. We expect that intermittent pulses should be stronger in this case. Hence we hope that this generalized model will be a useful tool for analysis of intermittent turbulence in space plasmas.

Naturally, the value of parameter $p$ (within some factor) is related to the usual models, which starting from Richardson's version of turbulence, try to recover the observed scaling exponents, which is based on the $p$-model of turbulence (e.g.,
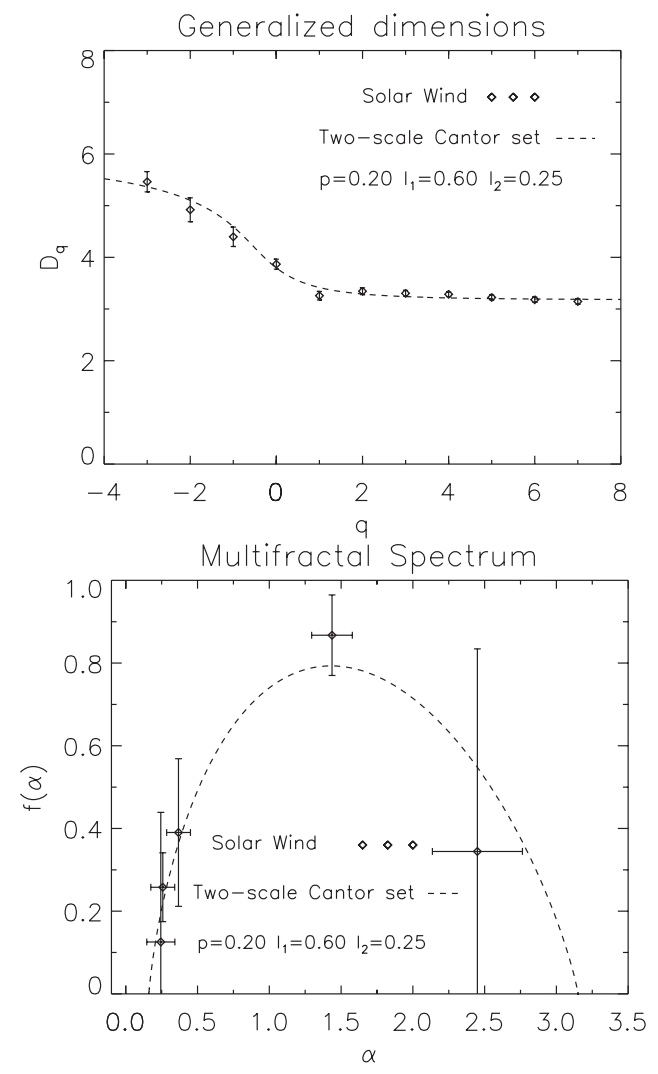

Fig. 4. (a) The generalized dimensions $D_{q}$ in Eq. (1) as a function of $q$. The correlation dimension is $D_{2}=3.4 \pm 0.2$ (see Table 1 ). The values of $D_{q}+3$ are calculated analytically for the weighted twoscale Cantor set with $p=0.20$ and $l_{1}=0.60, l_{2}=0.25$ (dashed line). (b) The singularity spectrum $f(\alpha)$ as a function of $\alpha$. The values of $f(\alpha)$ projected onto one axis for two-scale Cantor set with the same parameters (dashed).

Meneveau and Sreenivasan, 1987). The value of $p=0.12 \mathrm{ob}-$ tained here is roughly consistent with the fitted value in the literature both for laboratory and the solar wind turbulence, which is in the range $0.13 \leq p \leq 0.3$ (e.g., Burlaga, 1991; Carbone, 1993; Carbone and Bruno, 1996; Marsch et al., 1996). One should only bear in mind that here we take probability measure for the solar wind attractor, which quantifies multifractal nonuniformity of visiting various parts of the attractor in the phase space, while the usual $p$-model is related to the solar wind turbulence cascade for the dissipation rate, which resides in the physical space.

\section{Conclusions}

We have shown that the multifractal spectrum of the solar wind attractor is consistent with that for the multifractal measure of the self-similar weighted baker's map corresponding to the generalized two-scale weighted Cantor set. The values of the parameters fitted demonstrate 
small dissipation of the complex solar wind plasma and show that some parts of the attractor in phase space are visited at least one order of magnitudes more frequently than other parts as illustrated in Fig. 5 of Macek (1998). We think that this more genaral model will be a useful tool for analysis of intermittent turbulence in space plasmas. In particular, taking two different scales for eddies in the cascade, one obtains a more general situation than in the usual $p$-model for fully developed turbulence. We expect that intermittency and multifractality should be stronger for asymmetric scaling and a somewhat better agreement with the solar wind data could be obtained.

Thus our results provide direct supporting evidence that the complex solar wind is likely to have multifractal structure. In this way, we have further supported our previous conjecture that trajectories describing the system in the inertial manifold of phase space asymptotically approach the attractor of low-dimension (Macek, 1998). One can expect that the attractor in the low-speed solar wind plasma should contain information about the dynamic variations of the coronal streamers. It is also possible that it represents a structure of the time sequence of near-Sun coronal fine-stream tubes, see Macek (1998) and references therein.

Acknowledgements. This work has been supported by the Polish Ministry of Science and Higher Education (MNiSzW) through Grant N N202 4127 33. The author thanks A. Szczepaniak for help in preparation of figures.

Edited by: T. Hada

Reviewed by: two anonymous referees

\section{References}

Albano, A. M., Muench, J., Schwartz, C., Mees, A. I., and Rapp, P. E.: Singular-value decomposition and the GrassbergerProcaccia algorithm, Phys. Rev. A, 38, 3017-3026, 1988.

Bruno, R., Carbone, V., Veltri, P., Pietropaolo, E., and Bavassano, B.: Identifying intermittent events in the solar wind, Planet. Space Sci., 49, 1201-1210, 2001.

Burlaga, L. F.: Multifractal structure of the interplanetary magnetic field: Voyager 2 observations near 25 AU, 1987-1988, Geophys. Res. Lett., 18, 69-72, 1991.

Burlaga, L. F.: Lognormal and multifractal distributions of the heliospheric magnetic field, J. Geophys. Res., 106, 15917-15927, 2001.

Carbone, V.: Cascade model for intermittency in fully developed magnetohydrodynamic turbulence, Phys. Rev. Lett., 71, 15461548, 1993.

Carbone, V. and Bruno, R.: Cancellation exponents and multifractal scaling laws in the solar wind magnetohydrodynamic turbulence, Ann. Geophys., 14, 777-785, 1996, http://www.ann-geophys.net/14/777/1996/.

Eckmann, J.-P. and Ruelle, D.: Fundamental limitations for estimating dimensions and Lyapunov exponents in dynamical systems, Physica D, 56, 185-187, doi:10.1016/0167-2789(92)90023-G, 1992.
Grassberger, P.: Generalized dimensions of strange attractors, Phys. Lett. A, 97, 227-230, 1983.

Grassberger P. and Procaccia, I.: Measuring the strangeness of strange attractors, Physica D, 9, 189-208, 1983.

Halsey, T. C., Jensen, M. H., Kadanoff, L. P., Procaccia, I., and Shraiman, B. I.: Fractal measures and their singularities: The characterization of strange sets, Phys. Rev. A, 33, 1141-1151, 1986.

Hentschel, H. G. E. and Procaccia, I.: The infinite number of generalized dimensions of fractals and strange attractors, Physica D, 8, 435-444, 1983.

Macek, W. M.: Testing for an attractor in the solar wind flow, Physica D, 122, 254-264, 1998.

Macek, W. M.: Multifractality and chaos in the solar wind, in: Experimental Chaos, edited by: Boccaletti, S., Gluckman, B. J., Kurths, J., Pecora, L. M., and Spano, M. L., 622, American Institute of Physics, New York, pp. 74-79, 2002.

Macek, W. M.: The multifractal spectrum for the solar wind flow, in: Solar Wind 10, edited by: Velli, R., Bruno, R., and Malara, F., 679, American Institute of Physics, New York, pp. 530-533, 2003.

Macek, W. M.: Modeling multifractality of the solar wind, Space Sci. Rev., 122, 329-337, doi:10.1007/s11214-006-8185-z, 2006.

Macek W. M. and Redaelli, S.: Estimation of the entropy of the solar wind flow, Phys. Rev. E, 62, 6496-6504, 2000.

Macek W. M., Bruno, R., and Consolini, G.: Generalized dimensions for fluctuations in the solar wind, Phys. Review E, 72, 017202, doi: 10.1103/PhysRevE.72.017202, 2005.

Macek W. M., Bruno, R., and Consolini, G.: Testing for multifractality of the slow solar wind, Adv. Space Res. 37, 461-466, doi:10.1016/j.asr.2005.06.057, 2006.

Mandelbrot, B. B.: Multifractal measures, especially for the geophysicist, in Pure and Applied Geophys., 131, Birkhäuser Verlag, Basel, p. 5-42, 1989.

Meneveau, C. and Sreenivasan, K. R.: Simple multifractal cascade model for fully developed turbulence, Phys. Rev. Lett., 59, 14241427, 1987.

Marsch, E. and Tu, C.-Y.: Intermittency, non-Gaussian statistics and fractal scaling of MHD fluctuations in the solar wind, Nonlin. Processes Geophys., 4, 101-124, 1997, http://www.nonlin-processes-geophys.net/4/101/1997/.

Marsch, E., Tu, C.-Y., and Rosenbauer, H.: Multifractal scaling of the kinetic energy flux in solar wind turbulence, Ann. Geophys., 14, 259-269, 1996, http://www.ann-geophys.net/14/259/1996/.

Ott, E.: Chaos in Dynamical Systems, Cambridge University Press, Cambridge, 1993.

Schwenn, R.: Large-scale structure of the interplanetary medium, in: Physics of the Inner Heliosphere, edited by: Schwenn, R. and Marsch, E., 20, Springer-Verlag, Berlin, p. 99-182, 1990.

Theiler, J.: Spurious dimension from correlation algorithms applied to limited time-series data, Phys. Rev. A, 34, 2427-2432, 1986.

Theiler, J., Eubank, S., Longtin, A., Galdrikian, B., and Farmer, J. D.: Testing for nonlinearity in time series: the method of surrogate data, Physica D, 58, 77-94, 1992.

Tu, C.-Y. and Marsch, E.: Evidence for a 'background' spectrum of solar wind turbulence in the inner heliosphere, J. Geophys. Res., 95, 4337-4341, 1990. 